\title{
Vasculopatía post trasplante cardíaco
}

\author{
Luis Sepúlveda M.
}

La Insuf ciencia cardíaca avanzada mantiene, a pesar de tratamiento farmacológico y no farmacológico, la presencia de síntomas que limitan la actividad, disminuyen la calidad de vida, obligan a hospitalizaciones frecuentes por descompensación y reduce la sobrevida de los pacientes que la sufren. En casos seleccionados el trasplante cardíaco es la opción terapéutica con mejor resultado ${ }^{1}$. A pesar de los avances en los fármacos inmunosupresores, el éxito a largo plazo del trasplante cardíaco es limitado debido al desarrollo de un tipo particular de enfermedad coronaria aterosclerótica, conocida como vasculopatía del injerto (VDI), que es la principal causa de fallo del injerto y muerte luego del primer año del trasplante ${ }^{2}$. Es una forma de ateroesclerosis acelerada de tipo concéntrico (más que excéntrica) y difuso (más que focal) de las arterias epicárdicas y transmurales del injerto. En etapas iniciales se observa proliferación intimal y en sus etapas tardías, estenosis luminal. Aunque la patogénesis exacta de la VDI se mantiene sin ser establecida, hay suf ciente evidencia que muestra que mecanismos inmunológicos operan en un entorno de factores de riesgo no inmunológicos. Los eventos inmunológicos constituyen los estímulos iniciales, causando lesiones y disfunción endotelial, alteraciones de la permeabilidad endotelial, con la consiguiente hiperplasia miointimal y aumento de la síntesis de la matriz extracelular. La acumulación de lípidos en las arterias del injerto es importante, con atrapamiento de lipoproteínas en el tejido subendotelial, a través de interacciones con proteoglicanos Habría una activación de células musculares lisas de la media vascular, las que migran a la íntima, proliferan, y elaboran citoquinas y proteínas de la matriz extracelular, lo que resulta en una disminución del lumen vascular ${ }^{3,4}$. Cabe hacer notar que la enfermedad coronaria trasmitida por el donante no acelera la progresión de la vasculopatía ni altera la sobrevida a largo plazo ${ }^{5}$.

\section{Presentación clínica}

La incidencia de VDI varía de acuerdo con el método diagnóstico utilizado y f uctúa entre $10 \%$ y $60 \%$ el primer año (diagnóstico por coronariografía o por engrosamiento intimal en el ultrasonido intracoronario) hasta $42 \%$ a los 5 años (angiografía coronaria) ${ }^{2}$. Debido a la denervación del corazón trasplantado, la VDI es clínicamente silenciosa, y la isquemia no suele ser evidente hasta que la enfermedad está muy avanzada. Habitualmente los pacientes no presentan angina de pecho, aunque en algunos pacientes puede demostrarse reinervación cardíaca y ellos pueden tener dolor torácico. La VDI puede presentarse como el hallazgo incidental de un infarto silente, como insuf ciencia cardíaca por compromiso de la función sistólica o como muerte súbita. Esta forma de presentación tan particular obliga a los diferentes equipos de trasplante a implementar protocolos de evaluación en búsqueda de VDI, de forma invasiva o no.

\section{Evaluación no invasiva}

Debido a que la angiografía y la ecografía intravascular son pruebas invasivas, que representan aumento de los riesgos para los pacientes, las técnicas no invasivas han sido evaluadas, a pesar de que la isquemia mediante prue-

\section{Correspondencia:}

Dr. Luis Sepúlveda Morales

Clínica Alemana.

Hospital Clínico de la Universidad de Chile.

Luis.sepulveda.m@gmail.com 
bas funcionales no suele producirse hasta que la enfermedad es avanzada.

Test De Esfuerzo: Pocos estudios han analizado el papel de la electrocardiografía de esfuerzo en el diagnóstico de la VDI por las limitantes del método en esta población: electrocardiograma basal anormal, incapacidad para alcanzar la frecuencia cardíaca máxima (denervación). Algunos estudios han mostrado una baja sensibilidad y especif cidad para VDI, de $21 \%$ y $77 \%$, respectivamente ${ }^{6,7}$.

Ecocardiograma De Estrés: Varias técnicas ecocardiográf cas han sido evaluadas para estudiar la presencia de VDI: de estrés con dobutamina, contrastada y la asociación con hiperhemia inducida por Adenosina. La ecocardiografía de estrés con dobutamina es la técnica más frecuentemente utilizada para la evaluación de la motilidad y la función ventricular izquierda. Tiene una sensibilidad del $70 \%$ al $80 \%$ comparado con la coronariografía. Un eco dobutamina normal sugiere un buen valor predictivo negativo, en tanto que el deterioro de la motilidad se asocia con un incremento del riesgo de eventos $(\mathrm{RR}=7.3)^{6,8}$.

Cintigrama Miocárdico: Diferentes series, utilizando Talio con dipiridamol o ejercicio han descrito una sensibilidad de $21 \%$ a $58 \%$ y especif cidad de $64 \%$ a $88 \%$ para diagnóstico de VDI. La presencia de grandes defectos reversibles se correlaciona con un aumento de la mortalidad cardíaca ${ }^{6,9}$.

Tomografía Computada Coronaria: Varios estudios con pequeñas series de pacientes han presentado una sensibilidad de $83 \%$ a $86 \%$ y especif cidad de $95 \%$ a $99 \%$ para VID. La mayor limitante para la tomografía coronaria es la elevada frecuencia cardíaca de los pacientes trasplantados, lo que puede disminuir la calidad del examen. Además, que el uso de medio de contraste puede inducir nefropatía. La angioTAC coronario, por el momento, no es recomendado como técnica para evaluar VDI ${ }^{6,10}$.

Resonancia Nuclear Magnética Cardíaca: La angiografía coronaria por RNM ha mostrado una baja sensibilidad para VDI. Mejores resultados se observan al realizar evaluaciones de la reserva de perfusión miocárdica con Adenosina ${ }^{6,11}$.

\section{Evaluación invasiva}

Coronariografía: La angiografía coronaria sigue siendo, a pesar de sus limitaciones, la herramienta más utilizada para el diagnóstico de VID, porque permite guiar la terapia, ayuda a determinar pronóstico y a predecir eventos adversos. Su principal problema es que el medio de contraste llena sólo la luz del vaso y no nos informa acerca de la estructura de la pared arterial.
La clasif cación de Stanford describe las lesiones según su morfología, dividiéndolas en lesiones de tipo A, B1, B2, y C. La lesión Tipo A, estenosis focal, tubular, aislada o múltiple. Lesión de tipo B1 mantiene la normalidad del diámetro proximal y aparición brusca de estenosis difusa, concéntrico distal; tipo B2, reducción gradual concéntrica del diámetro vascular. Lesión tipo C vaso enfermo difusamente irregular, que ha perdido sus ramas y que termina abruptamente. De esta forma la angiografía detecta la presencia de vasculopatía en $10 \%$ a $20 \%$ de los pacientes trasplantados al año de seguimiento, y $50 \%$ a los 5 años. La presencia de estenosis angiográf camente signif cativa predice un mal pronóstico. En pacientes con al menos una estenosis focal de $40 \%$, la sobrevida fue de $67 \%$ a 1 año, $44 \%$ a los 2 años, y $17 \%$ a los 5 años. Con enfermedad de 3 vasos la sobrevida disminuye a $13 \%$ a los 2 años ${ }^{12,13}$.

Recientemente la Sociedad Internacional de Trasplante de Corazón y Pulmón (ISHLT) recomendó una nueva nomenclatura para la enfermedad vascular del injerto que está descrita por el Dr. Torres en su artículo ${ }^{14}$. Lo importante es el hecho que esta clasif cación predice un riesgo de muerte y retrasplante como resultado de VDI a 5 años de $7 \%$, que se eleva a $50 \%$ en los pacientes con enfermedad severa. Enfermedad rápidamente progresiva, def nida como la aparición de una lesión $>70 \%$ en un año predice un pronóstico aún peor ${ }^{6}$.

Ultrasonido Intravascular (IVUS): Esta técnica permite obtener una imagen transversal de alta resolución del lumen y de la pared vascular, lo que permite medir el grosor intimal y su composición. IVUS puede diagnosticar VDI en sitios aparentemente normales por angiografía, incrementando su incidencia a $75 \%$ al año $(10 \%$ por angiografía). Según IVUS se considera VDI cuando el grosor de la íntima es $>0.3 \mathrm{~mm}$ o cuando la suma del grosor de la íntima más la media es $>0.5 \mathrm{~mm}$.

La tasa de complicaciones por IVUS es baja, $1 \%$ a $3 \%$, describiéndose espasmos transitorios, disección u oclusión del vaso. Las principales limitaciones de la técnica son el tamaño del dispositivo, que permite evaluar sólo los troncos principales; el costo y el prolongado análisis de post procedimiento ${ }^{6}$.

Torres et al. ${ }^{14}$ conf rman la baja sensibilidad de la coronariografía v/s la coronariografía con IVUS en el diagnóstico de VDI. Encontraron una sensibilidad de $30.4 \%$ y una especif cidad de $92.3 \%$ para coronariografía cualitativa y de $52.1 \%$ y $69.2 \%$ para la coronariografía cuantitativa, en ambos casos comparado con IVUS. Resultados en todo similares a los descritos previamente ${ }^{13}$.

A pesar de sus reconocidas ventajas en el diagnóstico de VDI, la ISHLT no recomienda la ecografía intravas- 
cular como método de control rutinario de los pacientes trasplantados.

\section{Tratamiento de la enfermedad vascular del injerto}

Actualmente no existe una terapia claramente def nida para el tratamiento de la VDI, pero algunas medidas pueden disminuir su incidencia y progresión. El control de los factores de riesgo cardiovascular es importante evitar el uso del tabaco, el manejo de la dislipidemia, en ocasiones provocada por la misma terapia anti rechazo. Las estatinas han demostrado benef cios debidos a propiedades inmunomoduladoras, no inmunológicos y antiinf amatorias, más allá de los efectos hipolipemiantes directos.

El tratamiento antihipertensivo puede ser efectuado con inhibidores de la enzima de conversión de la angioten- sina, antagonistas de los receptores de angiotensina II o bloqueadores de los canales de calcio, como el diltiazem. Modif caciones de la terapia inmunosupresora: El uso de inmunosupresores como micofenolato o Inhibidores de la mTOR han demostrado disminuir la incidencia de VDI comparado con azatioprina. Si se diagnostica enfermedad vascular del injerto, se puede optar por cambios en la inmunosupresión e introducir inhibidores de la mTOR (sirolimus o everolimus) que han demostrado prevenir o enlentecer la progresión de la enfermedad vascular del injerto.

Si las lesiones coronarias son signif cativas y susceptibles de revascularización (lesiones focales) es posible efectuar revascularización percutánea con implante de stent o quirúrgica. En casos seleccionados el retrasplante cardíaco es la única alternativa terapéutica contra VDI ${ }^{15}$.

\section{Referencias:}

1. MEHRA MR, KOBASHIGAWA J, STARLING R, RUSSELL S, UBER PA, PARAMESHWAR J, et al Listing Criteria for Heart Transplantation: International Society for Heart and Lung Transplantation Guidelines for the Care of Cardiac Transplant Candidates 2006. J Heart Lung Transplant 2006; 25:1024-42.

2. STEHLIK J, EDWARDS LB, KUCHERYAVAYA AY, AURORA P, CHRISTIE JD, KIRK R, et al (2010). "The Registry of the International Society for Heart and Lung Transplantation: Twenty-seventh off cial adult heart transplant report-2010." The Journal of Heart and Lung Transplantation 29: 1089-1103.

3. RAHMANI M, CRUZ RP, GRANVILLE DJ, MCMANUS BM.. Allograft Vasculopathy Versus Atherosclerosis. Circ Res. 2006; 99:801-815.

4. TSUTSUI H, ZIADA KM, SCHOENHAGEN P, IYISOY A, MAGYAR WA, CROWE TD, et al. Lumen Loss in Transplant Coronary Artery Disease Is a Biphasic Process Involving Early Intimal Thickening and Late Constrictive Remodeling Results From a 5-Year Serial Intravascular U1trasound Study. Circulation. 2001; 104:653-657.

5. LI H, TANAKA K, ANZAI H, OESER B, LAI D, KOBASHIGAWA JA, et al. Inf uence of Pre-Existing Donor Atherosclerosis on the Development of Cardiac Allograft Vasculopathy and Outcomes in Heart Transplant Recipients. J. Am. Coll. Cardiol. 2006; 47;2470-2476

6. MEHRA MR, CRESPO-LEIRO MG, DIPCHAND A,
ENSMINGER SM, HIEMANN NE, KOBASHIGAWA JA, et al. International Society for Heart and Lung Transplantation working formulation of a standardized nomenclature for cardiac allograft vasculopathy-2010. J Heart Lung Transplant 2010; 29:717-727.

7. SMART FW, BALLANTYNE CM, COCANOUGHER B, FARMER JA, SEKELA ME, NOON GP, et al. Insensitivity of noninvasive tests to detect coronary artery vasculopathy after heart transplant. Am J Cardiol. 1991; 67:243.

8. SPES CH, KLAUSS V, MUDRA H, SCHNAACK SD, TAMMEN AR, RIEBER J, et.al. Diagnostic and prognostic value of serial dobutamine stress echo-cardiography for noninvasive assessment of cardiac allograft vasculopathy: a comparison with coronary angiography and intravascular ultrasound. Circulation. 1999; 100:509 -515.

9. CARLSEN J, TOFT JC, MORTENSEN SA, ARENDRUP $\mathrm{H}$, ALDERSHVILE J, HESSE B. Myocardial perfusion scintigraphy as a screening method for signif cant coronary artery stenosis in cardiac transplant recipients. J Heart Lung Transplant. 2000; 19:873-878.

10. ROMEO G, HOUYEL L, ANGEL CY, BRENOT P, RIOU JY, PAUL JF. Coronary stenosis detection by 16-slice computed tomography in heart transplant patients: comparison with conventional angiography and impact on clinical management. J Am Coll Cardiol. $2005 ; 45: 1826-1831$.

11. CAUS T, KOBER F, MARIN P, MOULY-BANDINI A, QUILICI J, MÉTRAS D, et al. Non-invasive diagnostic of cardiac allograft vasculopathy by $31 \mathrm{P}$ magnetic resonance 
chemical shift imaging. Eur J Cardiothorac Surg. 2006; 29 : 45- 49 .

12. GAO SZ, ALDERMAN EL, SCHROEDER JS, SILVERMAN JF, HUNT SA. Accelerated coronary vascular disease in the heart transplant patient: coronary arteriographic f ndings. J Am Coll Cardiol 1988; 12:334-40.

13. COSTANZO MR, NAFTEL DC, PRITZKER MR, HEILMAN JK 3RD, BOEHMER JP, BROZENA SC, et al. Heart transplant coronary artery disease detected by coronary angiography: A multi-institutional study of preoperative donor and recipient risk factors. J Heart Lung Transplant 1998; 17: 744-53.

14. TORRES H, ANINAT M, BECERRA L, MERELLO L, RAMOS S, MORA A, et al. comparación de angiografía coronaria con el ultrasonido intracoronario como gold standard en el diagnóstico de vasculopatía post trasplante cardiaco. Rev Chil Cardiol 2011: 30; 47 - 51.

15. MEHRA M. Contemporary concepts in prevention and treatment of cardiac allograft vasculopathy. AM J Transplant 2006; 6: 1248-56 